\title{
Satellite Detection of Moving Vessels in Marine Environments
}

\author{
Natalie Fridman, ${ }^{*}$ Doron Amir, ${ }^{*}$ Yinon Douchan, ${ }^{*}$ Noa Agmon ${ }^{* *}$ \\ ${ }^{*}$ Research \& Innovation Team \\ ImageSat International (ISI) \\ ** Computer Science Department \\ Bar-Ilan University
}

\begin{abstract}
There is a growing need for coverage of large maritime areas, mainly in the exclusive economic zone (EEZ). Due to the difficulty of accessing such large areas, the use of satellite based sensors is the most efficient and cost-effective way to perform this task. Vessel behavior prediction is a necessary ability for detection of moving vessels with satellite imagery. In this paper we present an algorithm for selection of the best satellite observation window to detect a moving object. First, we describe a model for vessel behavior prediction and compare its performance to two base models. We use real marine traffic data (AIS) to compare their ability to predict vessel behavior in a time frame of between 1-24 hours. Then, we present a KINGFISHER, maritime intelligence system which uses our algorithm to track suspected vessels with satellite sensor. We also present the results of the algorithm in operational scenarios of the KINGFISHER.
\end{abstract}

\section{Introduction}

There is a growing need for coverage detection of large maritime areas, mainly in the exclusive economic zone (EEZ). Physically accessing such a large area is practically impossible, thus the use of satellite-based sensors is an efficient and cost-effective way to perform this task. We focus on maritime missions, where the main goal is to monitor moving vessels with satellite sensors, in order to detect vessels that may mislead about their identity and transmit wrong identification parameters, for example their size and type. All civilian ships are required to transmit an Automatic Identification System (AIS) signal, which indicates the vessel's position and identification parameters. However, since this information is unreliable, the total maritime awareness is achieved through the integration and analysis of visual data from Electro-Optic (EO) and SAR satellites, enabling visual identification of vessels instead of relying only on cooperative vessel AIS transmission.

Satellite imagery is an expensive product, which covers a very small area, and can be acquired only at predefined acquisition opportunities. The time it takes from vessel transmission of an AIS signal until it can be analyzed in ground segment (known as data cycle), is typically over one

Copyright (C) 2019, Association for the Advancement of Artificial Intelligence (www.aaai.org). All rights reserved. hour. On the other hand, current commercial satellites mission planning may take several hours. Therefore, in order to task a satellite for detecting a moving vessel with no additional aids after 3 hours, one must cover a very large area of about $17,000 \mathrm{~km}^{2}$. This will end up in a cost of about $150,000-200,000$ USD per ship, which is practically not affordable. Choosing relevant satellite imagery is equivalent to looking for a needle in a haystack: one satellite image is commonly of size $50 \mathrm{~km}^{2}-100 \mathrm{~km}^{2}$, i.e., less than $1 \%$ of the total area's size. Therefore, in this paper we focus on efficient ways for decreasing the search area for the vessel by using vessel behavior prediction, and use that to increase the probability of acquiring relevant information from the satellite imagery. Reduction of the position uncertainty will also lead to a much more economic solution.

Despite significant progress in vessel prediction in maritime domain, existing solutions do not yet account for longterm vessel behavior prediction, thus they are irrelevant in the space domain. Our goal is to track moving vessels with satellite sensors, and select the most suitable satellite and its best time frame for detection (referred to observation window) for this mission. Thus, an algorithm for long-term prediction of vessel behavior is required in this domain.

In this paper we present an algorithm for selection of the best satellite observation window for detection of a moving object. First, we present a model for vessel behavior prediction and compare its performance to the base model. We use real marine traffic data (AIS) to compare the ability of the models to predict vessel behavior in a time frame of 1-24 hours. Based on satellite simulation, we propose a method for selecting the satellite observation window that increases the probability to detect the moving object.

Then, we present KINGFISHER, a maritime multilayered intelligence system created for an in-depth analysis of large maritime areas of interest, in order to provide maritime awareness and detect illegal activities such as illegal fishing. KINGFISHER uses the proposed algorithms to enable decision support for maritime operators and provide an optimal, autonomous and economic solution for maritime environments. Finally, we present the results of our algorithm on several operational scenarios. 


\section{Related Work}

Recently there has been an increasing interest in maritime awareness (Thomas 2006; Snidaro, Visentini, and Bryan 2015). While most of the research concentrates on anomalous behavior detection or event recognition (Riveiro et al. 2009; Lane et al. 2010; Roy 2009), predicting vessel behavior is still a big challenge for researchers. There are several approaches that were taken to tackle this challenge (Rhodes, Bomberger, and Zandipour 2007; Ristic et al. 2008; Tun et al. 2007), however, to the best our knowledge, existing models do not yet account for long-term vessel behavior prediction, thus they are irrelevant in the space domain.

Moreover, existing models assume that the destination of the vessel is known based on AIS message. In reality, destination field in AIS message is not always updated, and sometimes it is even falsely updated. Thus, in real application we cannot base our solution on reliability of destination field in AIS signal. Our goal is to develop a real service that can be offered by an industrial company to its clients. Additionally, we would like to have the ability of prediction to work freely, without the constant need for destination knowledge.

Rhodes et al. (Rhodes, Bomberger, and Zandipour 2007) use Artificial Neural Networks for predicting vessel behavior. They develop a model that learns the normal behavior of the vessel based on AIS data and enables detection of anomalies, and predicts the motion of the vessel in 15minute windows.

Ristic et al. (Ristic et al. 2008) develop an algorithm for motion prediction which is based on statistical analysis of AIS data. They extract motion patterns from historic data and use Kernel Density Estimation (KDE) to predict the vessel's motion. The results show prediction for 10, 30 and 70 minutes which again is too short for our domain.

A statistical approach was used also by Tun et al. (Tun et al. 2007). They propose to break the vessel's motion paths into separate regions using clustering techniques, and then learn the motion patterns using Hidden Markov Model (HMM). Tun et al. concentrate on high level activity, the movement of the vessel from one region to another, and propose to use their algorithms to detect abnormal vessel behavior.

Pallotta et al. (Pallotta, Vespe, and Bryan 2013) proposed a methodology called TREAD (Traffic Route Extraction and Anomaly Detection) for maritime situational awareness. They also used a statistical approach and unsupervised learning for vessel motion representation, and by detecting low-likelihood behavior, they enable the prediction of the vessel's future position. As in previous approaches, the proposed model does not account for long term prediction thus it is irrelevant in the space domain.

Despite significant progress in the satellite domain, many existing algorithms concentrate on mission planning and coverage of static targets such as area-of-interest or a specific location. Thus, the selection of necessary satellite imagery for analysis is based only on the area and time of interest. For example, Gao et al. (Gao, Wu, and Zhu 2013) propose to use ant colony optimization with the iteration local search algorithm to produce high quality schedules.
Other research direction treats dynamic targets in satellite domain, however mostly as image processing analysis, and the goal is to track moving object from an existing set of satellite imagery that was taken from nearby area and time. Meng and Kerekes (Meng and Kerekes 2012) present an object tracking algorithm from time-series multi-temporal and multi-spectral satellite images. Their results show that usage of both spectral and spatial feature ensures better tracking accuracy than using each of them alone.

Li et al. (Li, Guo, and Zhu 2011) propose a planning method for electronic and imagery satellites to work collaboratively for searching mission of moving ship. Their research shows that usage of electronic satellites to detect the vessel is necessary in searching mission.

Our goal is to track moving vessels with satellite sensors and select the best satellite observation window for this mission. In this paper, we present a model for long term prediction (up to 24 hours) of vessel behavior, which is required in this domain and show its usage in operational scenarios.

\section{Satellite Detection of a Moving Object - Framework}

Observation satellites have a limited window of opportunity for imaging of a given target area. In low earth orbit, a ground object will be in view for a few minutes at most. Detection of a moving object by satellite sensors is a challenging problem which requires a large amount of satellite imageries to enable vessel detection. We propose to minimize this coverage area by using a prediction process of the moving target

Figure 1 presents the movement of all vessels in a bounded area along seven days. The movement of vessels is received in the form of AIS data, and reflects 50000 AIS samples. We can clearly observe the created lanes of vessel movement, which provides a motivation for using graph representation for vessel behavior prediction, leading to a decrease in the imaging space.

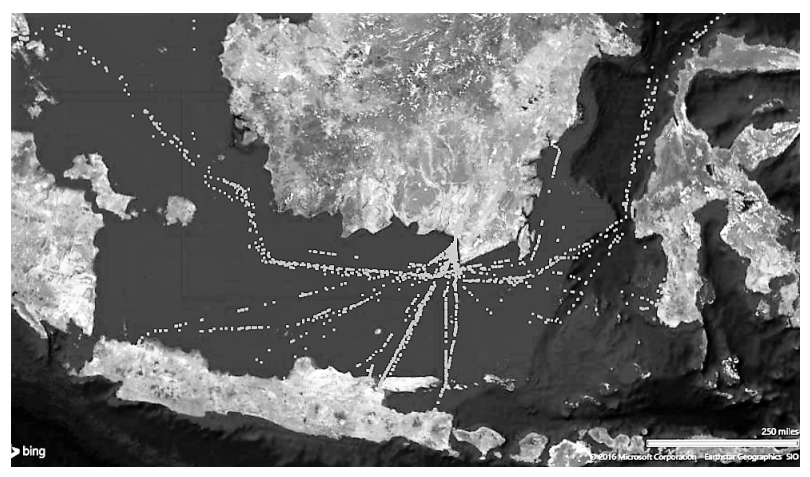

Figure 1: Actual vessels AIS samples over 7 days.

Let $S=\left\{s_{0}, \ldots, s_{m-1}\right\}$ be a set of $m$ satellites, each satellite $s_{i}$ has its set of observation windows $O W^{i}$. Our goal is to calculate the best opportunity to detect the moving target $x$ with one of the satellites in $S$. The process is described in Algorithm 1, which is executed for each tar- 
get $x$ that we wish to detect. In the following, we provide a high-level description of the algorithm, before examining each component in depth in the next sections.

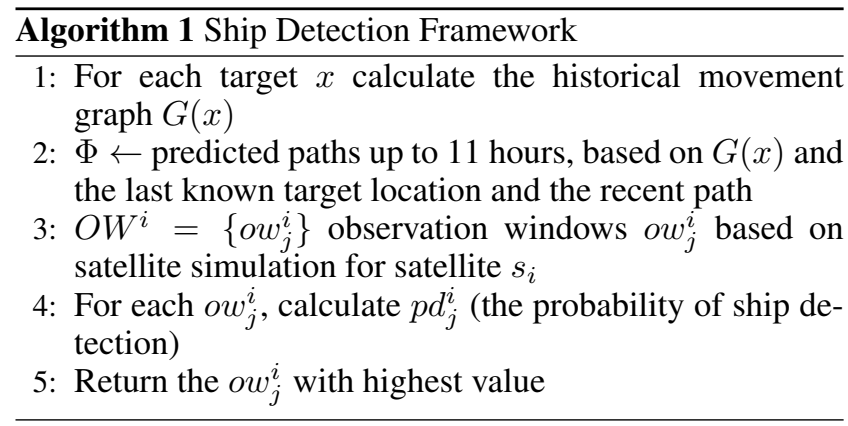

In line 1 , we calculate the historical graph $G(x)$ which represents all the historical movements of the target $x$. We use a stochastic model, Second Order Markov Chain, to represent all the historical movements of the target. Section 4.4 provides a detailed explanation regarding the graph construction.

Then, based on $G(x)$ and the last known location of the target and its recent path, we calculate the possible future paths, with respect to a motion model through each path. $\Phi$ gets the set of predicted future locations and their probability.

In line 3 , for each satellite $s_{i}$, we calculate all the observation windows $O W^{i}$ based on satellite simulation (Satellite Tool Kit-STK (STK )), that intersects with the predicted paths in $\Phi$. The STK simulation and the calculation of the observation windows are explained in depth in Section 5.

In line 4 , for each observation window $o w_{j}^{i}$ that corresponds to one of the satellites $s_{i} \in S$, we calculate the probability for detection $p d_{j}^{i} \in[0,1]$, which corresponds to the probability for the moving object to reach a specific location and the probability that this object can be detected at this location by satellite $s_{i}$. Let $p l_{j}^{i}$ be the probability of the target to reach to the geographical area $j$, it is calculated based on historical graph which represents all the paths and velocities. Let $p v_{j}^{i}$ be the probability of the target to be viewed at this location $j$ by satellite $s_{i} . p v_{j}^{i}$ is calculated based on parameters such as sensor ability, the target visibility and weather conditions. Thus, the probability $p d_{j}^{i}$ is defined as:

$$
p d_{j}^{i}=p l_{j}^{i} \times p v_{j}^{i}
$$

Note that in the our empirical evaluation, $p v_{j}^{i}=1$, i.e., we assume that if the target is present at a certain location, the satellite will necessary detect it. The reason for this is that we have examined information only from clear skies and we set the scan-width to be such that it attains this.

In line 5 we select the observation window $o w_{j}^{i}$ with the highest value for detecting the moving target.

\section{Model of Vessel Behavior Prediction}

Predicting vessel behavior is an important factor for detection of a moving vessel. Based on historical data of the vessel (AIS data), we create a behavioral model. We use Second
Order Markov Chain to build a graph representing the historical behavior. Based on the built graph and the estimation of the current state, we enable the prediction of future vessel locations. Figure 2 present AIS raw data of one of the vessel's motion during 13 months, and Figure 3 presents the graph constructed from this raw AIS data.

In this section we explore AIS data (Section 4.1), provide a detailed explanation regarding the preprocessing stage and graph construction (Section 4.2) and the vessel behavior prediction (Section 4.4).

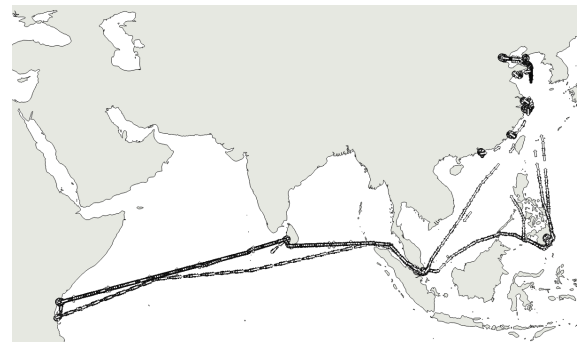

Figure 2: Vessel motion: Raw AIS data

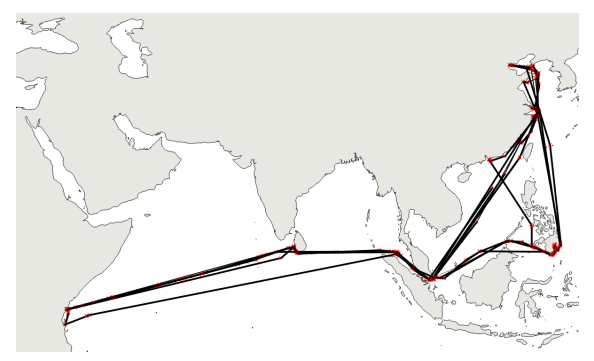

Figure 3: Vessel motion: Constructed graph

\subsection{AIS data}

Each AIS message contains unique identification of the vessel, and static and dynamic properties. Vessels transmit an AIS message every 1-5 minutes, depending on property type. This data is collected from both satellite and internetconnected shore-based stations, and can be available through a number of service providers. Due to a huge amount of data that AIS collectors should receive, which is filtered, and the fact that there are areas with low coverage rate, in practice we can obtain AIS signals of a specific vessel between once every two minutes and up to once every few hours.

The vessel dynamic data is calculated automatically using the internal equipment. It includes fields such as location, speed over ground (SOG), course over ground (COG) and others, and it is considered as relatively reliable data. Static data contains information about vessel properties such as destination, name, type, and dimensions, which are manually updated by the pilot, and are considered unreliable, as those are usually not up-to-date or contain missing values. Therefore, there is an inherent uncertainty in the information contained in AIS signals, thus the model of the vessel's behavior is noisy even before possible inaccuracies arise from fitting the data into a model. 


\subsection{Graph Creation: Second Order Markov Chain}

The AIS information may often be noisy and insufficiently frequent which challenge the behavior modeling process, and thus requires a preprocessing stage to enable further data transformation, such as graph representation. Therefore we interpolate data with spatial methods such as Slerp (Shoemake 1985).

Then, we divide the set of AIS signals, $D=$ $\left\{d_{0}, \ldots, d_{m-1}\right\}$, into leg segments $\Gamma=\left\{L_{0}, \ldots, L_{N-1}\right\}$. A leg segment corresponds to the vessels voyage as motion through straight lines. We partition the AIS signals by the turning points with heading direction changing over a threshold (over 10 degrees). This threshold is defined empirically and based on marine expert knowledge.

For example, as demonstrated in Figure 4, consecutive AIS signals partition the signal set into leg segments $L_{1}, L_{2}$, and $L_{3}$ where there is a course difference of more than 10 degrees between them.

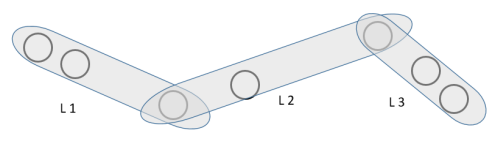

Figure 4: Preprocessing: creating legs

Then, we transfer the set of leg segments $\Gamma$, into a directed second order Markov chain (graph). The nodes and edges are calculated based on clustering and merging of leg segments vertices. The probability of transition between nodes is calculated based on historical behavior of the vessel.

Markov chains are stochastic processes that can be parameterized by empirically estimating transition probabilities between discrete states. It can be used for describing systems that follow a chain of linked events, where what happens next depends only on the current state(s) of the system: in Markov chain of the first order, each subsequent state depends only on the current state. In Markov chains of second or higher orders, the next state depends on two or more predecessor states.

Each node $N_{i}$ in the graph corresponds to a physical location in the world, and an edge between $N_{i}$ and $N_{j}$ is a possible transition between the states. Nodes are calculated as clusters of geographic legs' vertices, and the clusters are calculated using the distance across the sphere (Haversine), where the condition of clustering is maximum distance. The maximal distance between samples in the same cluster is set with a predefined value $(15 \mathrm{~km}$, defined empirically and based on marine expert knowledge). The Edges of the movement graph $G(X)$ are the transitions described by Leg segments with vertices in the same created nodes. Each edge is parametrized with a conditional probability for preforming the corresponding transition, given the previous transition.

For example, as shown in Figure $5, N_{1}, N_{2}$ and $N_{3}$ are the nodes of the graph which is created by clustering of relatively close leg segment vertices.

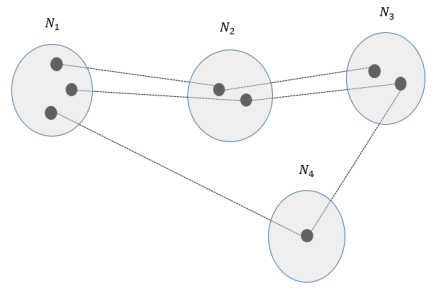

Figure 5: Graph creation

In the First Order Markov Chain model, each edge in the graph contains the probability of the vessel to travel from one node to another, and it is calculated based on statistical analysis of leg segments that passes through these two nodes. Due to unreliability of the destination field in the AIS signal, which is often not up-to-date, we use Second Order Markov Chain for vessel behavior prediction. Thus, each edge represents three vertices. For example, edge (a,b,c) corresponds to the state transition of $N_{a} \rightarrow N_{b} \rightarrow N_{c}$, and the corresponding probability $P\left(T_{n}=N_{b} \rightarrow N_{c} \mid T_{n-1}=\right.$ $N_{a} \rightarrow N_{b}$ ), is the probability to preform transition $T_{n}$ given the previous transition $T_{n-1}$ has occurred.

For example, as shown in Figure 6 the probability on each edge between $N_{1}$ and $N_{2}$ is calculated based on predecessor of $N_{1}$ which in our example is $N_{0}$. If there are several predecessors for the node, as in $N_{6}$, then the probability is calculated based on each option. In our example, there are two edges that connect $N_{6}$ and $N_{8}$, one for each predecessor $\left(N_{3}\right.$ and $\left.N_{2}\right)$ and the probability is calculated accordingly, $P(3,6,8)$ and $P(2,6,8)$.

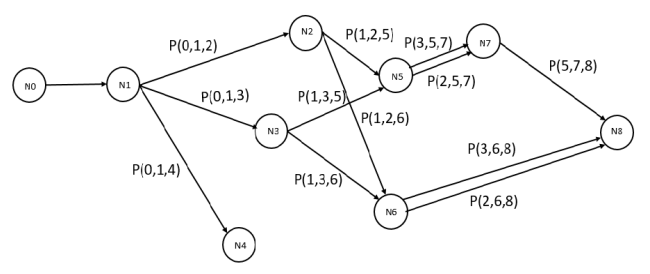

Figure 6: Example of a Second Markovian representation of our problem.

Each edge in the graph corresponds to movement of the vessel through two geographical locations (nodes in the graph). There are edges that represent long distances of $600 \mathrm{~km}$ and more between pairs of nodes, and the velocity changes along the edge. Therefore, in order to provide a more accurate prediction of the movement, the possible vessel velocities on each edge are partitioned into a set of speed segments. Each velocity sample along the edge that exceeds a velocity change threshold, is marked to represent a new speed segment and for each speed segment statistics are measured upon the distribution of the relevant velocity values of: minimum, maximum, mean and standard deviation of the vessel. 


\subsection{Setting the current state}

In order to enable the behavior prediction, first we need to set the current state of the vessel on the movement graph. Let $D=\left\{d_{0}, \ldots, d_{k}\right\}$ be a set of AIS signals, representing the current path of the vessel and $G(x)$ is the movement graph. Our goal is to identify the current location in the movement graph $G(x)$ that represents the current vessel position $d_{k}$.

AIS signals come in different time intervals, and the data is noisy and may include missing signals, thus trivial solution such as selecting the closest node to $d_{k}$ is not suitable for this domain. For example, the closest node in $G(x)$ to $d_{k}$ may not be a part of the vessel's route, thus our solution should take the vessel's recent movement into account.

Our solution is to match the vessel's route to the nodes in graph $G(x)$. First we interpolate the current vessel path $D$, to overcome on the missing signals and to improve the matching process. Then, from the interpolated $D$, we select a set of representative signals $D^{\prime}$ which are close to the nodes in $G(x)$. For each pair in signal set $D^{\prime}$ we search the possible edges in graph $G(x)$, and the set of connected nodes represent a possible edge in the graph. We rank each route based on the distance between the signals in set $D^{\prime}$ to the possible nodes in $G(x)$ and also the distance between $d_{k}$ to the corresponding edge. The probability for current location candidates are ascribed according to the computed rank.

\subsection{Behavior Prediction}

Based on the historical graph $G(x)$ and the initial vessel's location on the graph, we extract the possible paths of the vessel at different times. Each such path contains set of edges with their speed segments and probabilities. Using multi agent simulation we predict the vessel behavior.

We create agents that simulate the possible movements of the vessel on the extracted paths. Each agent gets the movement path based on path's probability, thus the higher the probability, the more agents will move on this path. Each agent draws different velocities according to the behavior graph, based on the Gaussian distribution of velocities on each edge. At any given time, the model return the positions of the agents. The predicted area of the vessel is a two dimensional histogram, which is created based on the agents' location. Each cell in the histogram represents geographic location $1 \mathrm{~km} \times 1 \mathrm{~km}$ and the rank of each cell is the normalized number of agents (based on the total number of agents).

\section{Satellite Simulation}

In this section we want to find set of the observation windows $O W^{i}=o w_{j}^{i}$ for each satellite $s_{i} \in S$ where each $o w_{j}^{i} \in O W^{i}$ intersects with the vessel's predicted paths $(\Phi)$ in both space and time, meaning that the moving vessel can be detected within $o w_{j}^{i}$.

We use an STK (Satellite Tool Kit) (STK ) as satellite simulation for moving object detection. STK is a physics based software package that allows to perform complex analysis for problems involving Earth-orbiting satellites. The STK simulation enables the calculation of the satellite-to-object visibility. Our simulation calculates the times that the satellites can acquire the simulated moving vessels based on position information and satellite constraints.
Based on the set of all predicted paths in $\Phi$ and its velocities, we create 10000 agents (vessels) moving along the predicted paths. The behavior of each such agent is set based on historical model behavior and the movement graph. All agents in our simulation are running simultaneously from the identical initial location.

Each observation window $o w_{j}^{i}$ is ranked based on normalized sum of visible agents by satellite $s_{i}$. Thus, let $A=\left\{a_{0}, \ldots, a_{n-1}\right\}$ be a set of $n$ agents, and $A V_{j}^{i}=$ $\left\{a v_{0}, \ldots, a v_{k-1}\right\}, A V_{j}^{i} \subseteq A$ is a set of visible agents by satellite $s_{i}$ in observation window $o w_{j}^{i}$. Thus the probability $p l_{j}^{i}$ of the vessel to be visible by satellite $s_{i}$ in observation window $o w_{j}^{i}$ is defined as following:

$$
p l_{j}^{i}=\frac{\left|A V_{j}^{i}\right|}{|A|}
$$

Figure 7 presents the STK simulation of an experiment, showing the agents moving on the created graph based on the historic statistics.

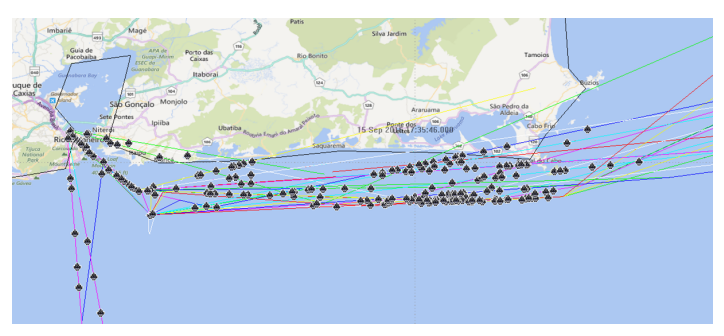

Figure 7: STK simulation

\section{Vessel Prediction in Maritime Domain}

In this experiment we wanted to compare the performance of our model to the base model, Straight-Line model. The Straight-Line model predicts the future position of the vessel according to its recent course and the speed. The predicted area of the Straight-Line model is a two dimensional histogram, which is created based on the Gaussian distribution around the predicted point.

We created two Straight Line models, Straight-LineConst and Straight-Line-Widen. The Straight-Line-Const sets the std of the distribution as a constant value, which is set to $2 \mathrm{~km}$ based on domain expert knowledge. The Straight-Line-Widen sets the std of the distribution based on the time of the prediction, which means the longer the predicted time the wider the predicted area is. In this case we significantly enlarged the predicted area based on the prediction time.

We randomly selected a set of vessels in different locations in the world. AIS Data of 24 months was collected for each vessel, where the first 18 months data was used for the extraction of historical graph model, and the additional 6 month was used for prediction validation.

For each experimental vessel, the data for prediction validation was divided into 10 test-subsets. Each test-subset is divided into two non-overlapping smaller subsets: a present 
subset, which is used for extracting the vessel current location in the movement graph, and a future subset which is used for prediction validation. We examined whether the prediction results in the time intervals of up to 24 hours corresponds to the future signal set.

The experiment was carried out using 100 vessels, 1000 test-subsets for prediction validation, from which test subsets were dropped in cases where failure has occurred in the procedure of setting the current state. The procedure can fail to set the vessel current state if it is on a new route, meaning that the current position of the vessel is too far from its historical routes. Our model predicts vessel behavior based on historical data, thus in cases where there is lack of historical data due to data provider reception or vessel transmission issues, other prediction approaches should be considered (we leave this for future work).

We compared the models with respect to two parameters: Hit-Percentage and Area-Ratio. We controlled the time of the prediction, 1-24 hours, and measured the success of the prediction based on the defined parameters.

Hit-Percentage parameter is the successful prediction rate for the vessel's locations. If the real location of the vessel was inside of the model's recommended polygons, then the prediction is successful.

Area-Ratio parameter indicates to area coverage reduction. It is calculated as the area that was recommended by the model divided by the area which should be covered without any prediction model (naive area). The naive area is calculated as $\pi r^{2}$ where $r$ is the distance with the maximum reasonable speed, 13 knots, multiplied by the prediction time. Lower ratio means more economic model.

Figure 8 displays the comparison of the models on HitPercentage parameter. The $\mathrm{x}$-axis represent the prediction time and the $y$-axis represent the percentage of successful prediction. The results show that our History Graph model (mean value: 0.67 ) has a much higher number of successful predictions than the Straight-Line-Const model (mean value: 0.47 ) and Straight-Line-Widen model (mean value: 0.60). Moreover, the History Graph model was found to be significantly higher in Hit-Percentage parameter compared to the Straight-Line-Const and Straight-Line-Wide models, with p-value $<0.01$ (using the ANOVA test).

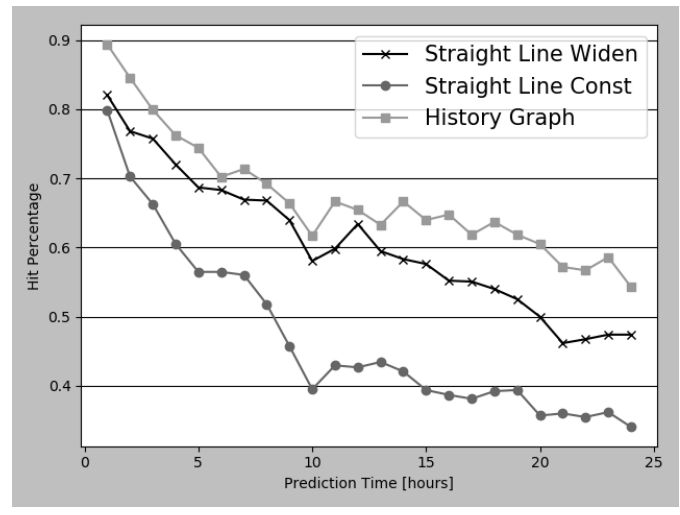

Figure 8: Hit-Percentage results
Figure 9 presents the comparison between the models on Area-Ratio parameter. The results show that StraightLine-Const model (mean value: 0.0008 ) has significantly lower Area Ratio rate than History-Graph model (mean value: 0.01) and Straight-Line-Widen model (mean value: 0.009 ) with $\mathrm{p}$-value $<0.01$. Moreover, the results show that the main difference between the Straight-Line-Const and History-Graph models is in short-term prediction (up to 4 hours), while in long term the models have similar values. Straight-Line-Widen has much higher values in long-termpredictions but it still has a lower Hit-percentage than the History-Graph which means that is not enough to enlarge the coverage area but also take into account the behavior pattern of the vessel.

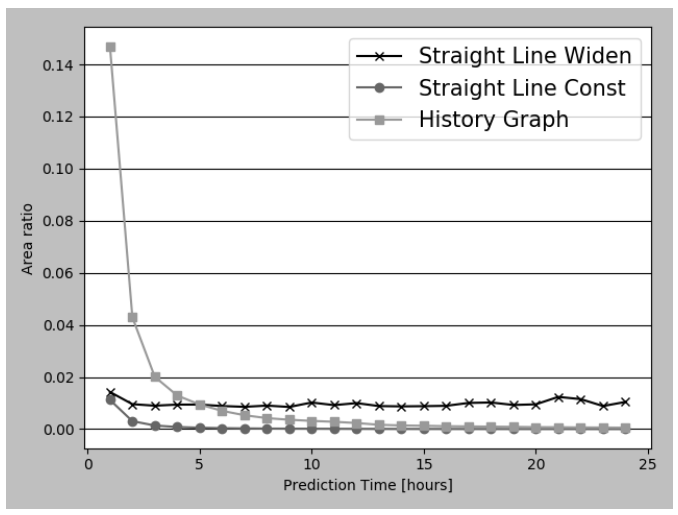

Figure 9: Area Ratio results

The results verify that the History-Graph model provides a more accurate prediction than both Straight-Line models. While the results show that Straight-Line-Const has better results in Area-Ratio parameter, in accuracy prediction it performed worst. History-Graph model has good results in prediction and reduces the required area coverage by more than $99 \%$ in comparison to area that should be covered without any prediction model.

\section{KINGFISHER: System Architecture}

The KINGFISHER is a maritime multi-layered intelligence system created for an in-depth analysis of large maritime areas of interest in order to provide maritime awareness and detect illegal activities. KINGFISHER is an operational system and is used in different scenarios and by dozens of users around the world.

The Research and Innovation team at ImageSat International (ISI) developed the KINGFISHER based on Artificial Intelligence (AI) techniques such as Multi Agent Simulation and Deep learning. The objective of the AI techniques is to enable decision support for maritime operators and provide an optimal, autonomous and economic solution for maritime system.

KINGFISHER integrates data from several types of satellite-based sensors and intelligence sources such as AIS, SAR satellite imagery, EO satellite imagery, OSINT, 
weather and more. Figure 10 presents the system architecture.

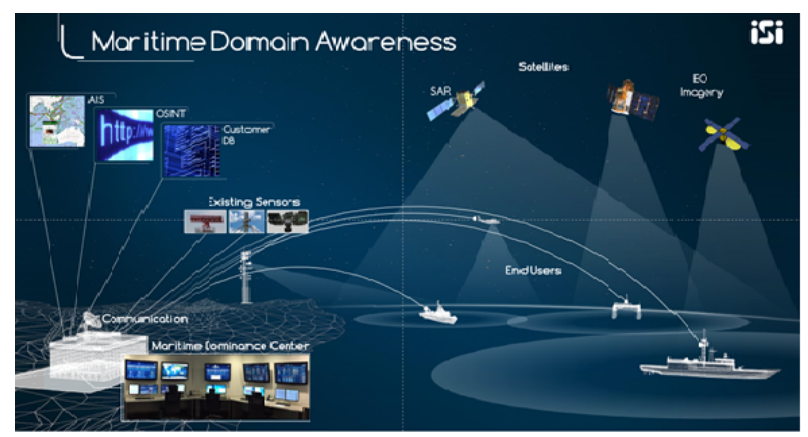

Figure 10: System architecture

\subsection{Algorithmic Framework and AI}

There are two main algorithmic phases at KINGFISHER. First is to continuously monitoring an area in order to detect and alert the operator about presence of maritime anomalies. Then, the system performs further investigation for all the suspected vessels to learn their behavior patterns and to continue tracking these vessels with satellite sensors, and the system recommends the best imaging opportunities available.

In the monitoring phase, the data from various sources is being received, analyzed and archived as a separate intelligence layer. Deep Learning algorithms are applied on the imagery data layers in order to autonomously detect vessels in the imagery, classify their type and identify specific vessels that are important to the operators. The system enables detection of naval vessels which are mostly non-cooperative targets through the integration and correlation of all data sources.

To continue to track the suspect vessels with satellite sensors, the vessels prediction and satellite selection algorithms are being used. It enables the operators to track the suspect a vessel for days or even weeks and incriminate it on illegal activities.

\section{Satellite detection of maritime vessels: Examples}

KINGFISHER is an operational system which is used by dozens of users around the world. While most of the operational usage of the system is classified by our clients, we will present here general examples of our own usage of the system, with no connection to any specific client.

In our first example, we have monitored a Russian research ship "YANTAR" sailing towards the Black Sea in September 2017 after completing its activities in the eastern Mediterranean. Our goal was to detect the vessel with a satellite sensor. Based on the known historical data of the vessel and its last known position, we have predicted its future location. We have targeted the best suited-satellite and time frame for this mission, and acquired a satellite image about 12 hours after its last known location. We set the satellite to capture 4 images, each image was $200 \mathrm{~km}^{2}$, thus in total we covered $800 \mathrm{~km}^{2}$, for 12 hours of uncertainty.
Figure 11 presents the uncertainty cycle (red circle) that was created in 12 hours based on the last known location of the vessel and the vessel mean speed. In 12 hours the vessel can be anywhere in the red circle. The white square represent the possible size of the satellite imagery. We can see that while one could possibly need thousands of satellite imageries in order to cover all the area, we use only 4 such images to detect the vessel.

Figure 12 presents the successful detection of the "YANTAR" with EROS B satellite. The figure shows the prediction results, the covered area by the EROS B satellite and the exact location of the "YANTAR" with the successful detection.

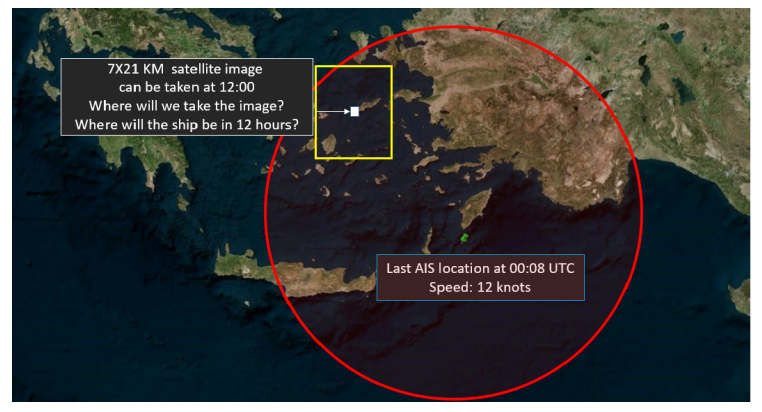

Figure 11: Uncertainty cycle after 12 hours

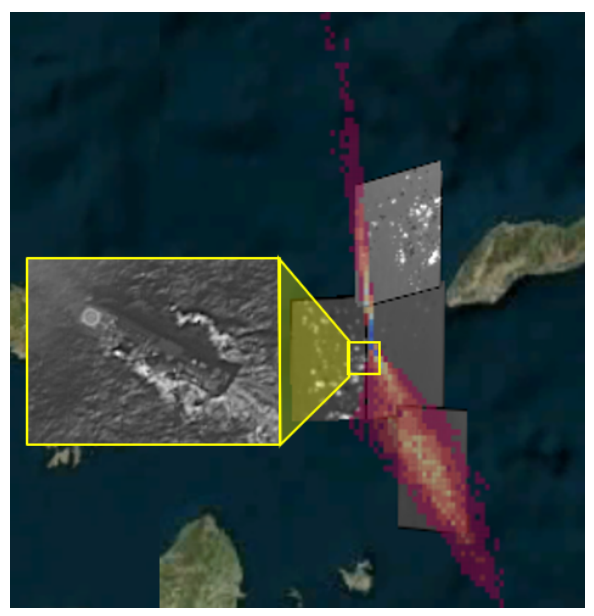

Figure 12: Successful detection of the YANTAR: Russian research ship

In our next example, we have monitored a submarine on its way from the Atlantic Ocean to the Mediterranean sea, on October 9, 2017. The submarine has no historical data, thus we used common shipping routes and historical data of other vessels in the area for graph creation. We used intelligence information to estimate the submarine last known location and its speed. We also estimated that the submarine will surface before crossing the Strait of Gibraltar. We predicted the location of the submarine based on the pass of the EROS B satellite in this area. A prediction was made for five hours. We used 3 satellite imagery and covered $600 \mathrm{~km}^{2}$ of the area. 
Figure 13 presents the successful detection of the submarine inside the Mediterranean sea. Moreover, in the satellite imagery we can see another military vessel, a corvette sailing close to the submarine.

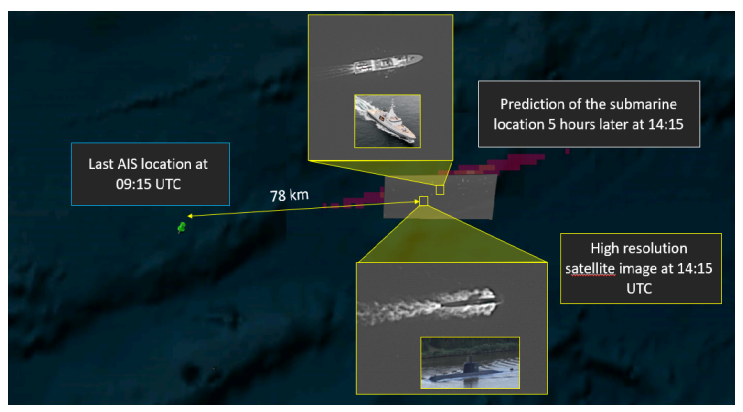

Figure 13: Successful detection of the Submarine

On October 10, 2017 the day after our first successful detection of the submarine in the Mediterranean sea, KINGFISHER succeeded again in detecting the submarine. Now, we used our first detection as the last known location of the submarine and set the EROS B satellite based on the prediction algorithm using the same graph as was built in our first try. Figure 14 presents the another successful detection of the submarine.

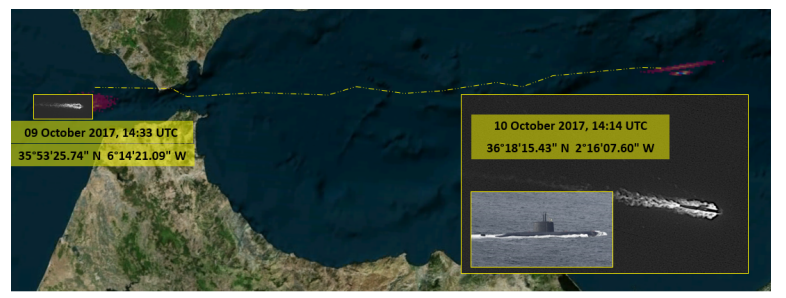

Figure 14: Another successful detection of the Submarine

\section{Summary and Future Work}

This paper presents an algorithm for selection of the best satellite observation window for detection of a moving object. Satellite imagery is an expensive product, which covers a small area, and can be acquired only at predefined acquisition opportunities. We focus on efficient ways for decreasing the search area for the moving vessel by using vessel behavior prediction, which increases the probability of acquiring relevant information from the satellite imagery.

We also presented KINGFISHER, maritime intelligence system which uses the proposed algorithms to track a maritime vessels with satellite sensors. We present three examples of successful detection based on the proposed algorithms.

In our future work, we plan to improve the prediction model to count for longer prediction times, for up to 48 hours decrease the recommended areas for vessel detection even more.

\section{References}

Gao, K. B.; Wu, G. H.; and Zhu, J. H. 2013. Multi-satellite observation scheduling based on a hybrid ant colony optimization. In Advanced Materials Research, volume 765, 532-536. Trans Tech Publ.

Lane, R. O.; Nevell, D. A.; Hayward, S. D.; and Beaney, T. W. 2010. Maritime anomaly detection and threat assessment. In In Proceedings of the 13th Conference on Information Fusion (FUSION), 1-8. IEEE.

Li, J.-J.; Guo, H.; and Zhu, J.-H. 2011. Planning method for multi-satellite cooperating search for a moving target on the sea. In In Proceedings of the International Workshop on Multi-Platform/Multi-Sensor Remote Sensing and Mapping (M2RSM), 1-6. IEEE.

Meng, L., and Kerekes, J. P. 2012. Object tracking using high resolution satellite imagery. IEEE Journal of Selected Topics in Applied Earth Observations and Remote Sensing 5(1):146-152.

Pallotta, G.; Vespe, M.; and Bryan, K. 2013. Vessel pattern knowledge discovery from ais data: A framework for anomaly detection and route prediction. Entropy 15(6):2218-2245.

Rhodes, B. J.; Bomberger, N. A.; and Zandipour, M. 2007. Probabilistic associative learning of vessel motion patterns at multiple spatial scales for maritime situation awareness. In Information Fusion.

Ristic, B.; La Scala, B.; Morelande, M.; and Gordon, N. 2008. Statistical analysis of motion patterns in ais data: Anomaly detection and motion prediction. In Information Fusion, 1-7. IEEE.

Riveiro, M.; Falkman, G.; Ziemke, T.; and Kronhamn, T. 2009. Reasoning about anomalies: a study of the analytical process of detecting and identifying anomalous behavior in maritime traffic data. In SPIE Defense, Security, and Sensing, 73460A-73460A. International Society for Optics and Photonics.

Roy, J. 2009. Automated reasoning for maritime anomaly detection. In In Proceedings of the NATO Workshop on Data Fusion and Anomaly Detection for Maritime Situational Awareness (MSA 2009), NATO Undersea Research Centre (NURC), La Spezia, Italy, 15-17.

Shoemake, K. 1985. Animating rotation with quaternion curves. In ACM SIGGRAPH computer graphics, volume 19, 245-254. ACM.

Snidaro, L.; Visentini, I.; and Bryan, K. 2015. Fusing uncertain knowledge and evidence for maritime situational awareness via markov logic networks. Information Fusion 21:159-172.

Satellite tool kit. https://www.agi.com/products/stk/.

Thomas, G. 2006. Maritime domain awareness. Coast Guard Journal of Safety \& Security at Sea, Proceedings of the Marine Safety \& Security Council 63(1).

Tun, M. H.; Chambers, G. S.; Tan, T.; and Ly, T. 2007. Maritime port intelligence using ais data. Recent advances in security technology 33. 